\title{
Cutaneous allergy
}

\author{
DA McKay \\ Consultant Dermatologist, Royal Infirmary of Edinburgh, Edinburgh UK
}

\begin{abstract}
Cutaneous allergy encompasses a broad spectrum of clinical presentations with complex underlying immunological mechanisms. Allergy may be considered to play a role in disorders that include atopic dermatitis, contact dermatitis, urticaria, angioedema, anaphylaxis and adverse drug reactions. The skin phenotypes associated with allergy are more commonly exhibited in non-allergic disease and so a high proportion of patients who consider themselves allergic in truth have other, nonallergic explanations for their presentation. Investigations may help to delineate those individuals who are more likely to have true allergy, but tests currently available have significant limitations and are open to easy misinterpretation. As a consequence, any investigations are best carried out by someone experienced in managing patients with potentially allergic disorders.
\end{abstract}

Correspondence to DA McKay

Department of Dermatology Lauriston Building

Lauriston Place

Edinburgh EH3 9HA, UK

tel. +44 (0) I 3 I 536244 I

e-mail davidmckay@nhs.net

KEYWORDS Food allergy, atopic dermatitis, urticaria, anaphylaxis, drug allergy

DECLARATION OF INTERESTS

\section{OVERVIEW}

Allergy encompasses a broad range of clinical symptoms that are all characterised by an acquired hypersensitivity to some sort of exogenous agent. In recent years, allergy, and in particular food allergy, has increased both in prevalence and diversity across the globe. This has led to the inevitable consequence that patients and clinicians alike will consider allergy as the primary stimulus for a number of different conditions, including skin disease. In reality, there is a complex but indistinct relationship between allergy and cutaneous disorders. Access to specialist centres that have the clinical knowledge and tools to investigate patients with suspected allergy might be limited, even in developed countries.

\section{DIAGNOSIS OF ALLERGY}

In general, investigations for potential allergy should be directed by a relevant clinical history. Randomly testing patients in the absence of a supporting history will rarely produce clinically meaningful results and may lead to patients being erroneously diagnosed with allergy. Where allergy is suspected, or needs excluding, there are a number of investigations that can be considered.

\section{Specific IgE measurement}

Atopy, a tendency to form immunoglobulin $E$ (IgE) antibodies in response to an antigenic, usually protein stimulus, encompasses a number of 'allergic' conditions that include atopic dermatitis, urticaria, food allergy, allergic rhinitis and asthma. Most immunology laboratories can directly measure serum-specific lgE levels to a wide range of suspected allergens. Positive tests indicate sensitisation, and in general, a higher level of specific IgE increases the likelihood of clinical allergy. Higher IgE levels, however, do not necessarily indicate the severity of any ensuing reaction. A high background total IgE in very atopic individuals can lead to falsepositive specific lgE levels, rendering the test difficult or impossible to interpret. In part due to variation in antigenic epitopes and because of the recognition of non-IgE-mediated allergic mechanisms, a negative result alone cannot reliably exclude allergy.

\section{Skin prick testing}

Skin prick testing is essentially a clinical test of lgE production and has the advantage that results are available immediately. Allergen droplets are gently scratched onto the skin, and a positive result is indicated by a wheal reaction within 15 minutes. In general, skin prick tests are more sensitive than specific serum IgE tests, but cannot be performed if patients are taking antihistamines (long-term oral corticosteroids do not affect skin prick test results). A negative skin prick test is reasonable evidence of absence of IgE-mediated allergy, but positive results need careful interpretation.

\section{Food diary}

Western diets are increasingly complex and varied. Patterns of potential food allergic reactions may be more easily discernible if a patient is able to keep an accurate food diary, which in turn may direct further investigations. 


\section{Food challenge}

Oral food challenge is considered the gold standard in testing for food allergy, but is only practicable in specialised allergy centres. Although an open food challenge, where the clinician and patient are both aware of the nature of the challenge substance may be necessary in some situations, either a single (patient) blinded or double (patient and clinician) blinded challenge are considered more reliable tests.

\section{Intradermal testing}

Intradermal testing is usually reserved for the investigation of certain types of drug allergy. Small volumes of initially highly diluted drug are injected into the dermis. The test is time consuming and carries with it a risk of systemic reaction, and so should only be performed in specialist centres with access to adequate resuscitation facilities.

\section{Patch testing}

Patients with suspected allergic contact dermatitis are investigated with patch testing. Typically a series of patches of suspected chemical allergens are applied to the patient's skin and remain in place for 48 hours. Any skin reaction is recorded when the patches are removed, and read again a further $\mathbf{4 8}$ hours later. Short contact testing, where allergens are applied to the skin for minutes can be used to investigate immediate contact reactions.

\section{Other tests}

Patients may have more ready access to other, commercially available tests such as Vega testing, hair analysis, applied kinesiology and specfic lgG levels. None of these have any robust scientific grounding to advocate their use - they are not helpful and may be counterproductive in the assessment and management of patients with allergic disease.

\section{SPECIFIC SKIN CONDITIONS}

\section{Atopic dermatitis}

Atopic dermatitis (AD) is a chronic relapsing inflammatory disorder characterised by intense itch, dry and inflamed skin. It is thought to occur as a consequence of gene-environment interactions that involve defective skin barrier function and immune dysregulation. A total of $80 \%$ of cases of $A D$ are associated with high lgE levels and peripheral eosinophilia. It typically presents in early childhood, but can persist or occur for the first time in adults. Up to $25 \%$ of children in Westernised countries are affected and the worldwide prevalence continues to rise. Although $A D$ varies in severity, it has been consistently associated with impaired quality of life measures such as sleep disturbance and problematic behaviour; psychological stress and depression; and disruption to family life. It is also associated with other atopic co-morbidities such as food hypersensitivity, hay fever and asthma (the 'atopic march').

\section{Food allergy and atopic dermatitis}

Defects in epidermal and gut barrier function may facilitate sensitisation to food allergens, and there is indisputable evidence that $A D$ is associated with such sensitisation. Clinical reactions to food include eczematous, non-eczematous and combined reactions which may involve IgE and non-lgE mediated mechanisms. Positive food challenges occur in $33-56 \%$ of infants with $A D$, but typically in highly selected populations referred to specialist allergy clinics. Cow's milk, hen's egg, soy, wheat, fish, peanut, or tree nut allergy account for $90 \%$ of cases of infants with $A D$ and food allergy. Eczematous reactions to food are more likely in severe, early onset $A D$, and will follow immediate reactions in over $40 \%$ of subjects. A recent Cochrane Review concluded there was no evidence for dietary exclusions in the treatment of established $A D$ in unselected populations. Food sensitisation therefore does not always equate to clinically relevant food allergy, but may be relevant for a subset of children and a minority of adults.

An approach to food allergy in $A D$ should consider factors such as age, severity, response to treatment, co-morbidities, availability of allergy services and not least patient expectation. Specialised allergy clinics have a higher expectation of identifying relevant food allergy in their selected populations, but this cannot be generalised to the larger population of $A D$ managed in primary care. Moreover, investigation and management of food allergy are not a substitute for good skin care.

\section{Aero-allergy and atopic dermatitis}

Sensitisation to aero-allergens occurs early in life, and those skin barrier defects implicated in food allergen sensitisation may play a similar role in aero-allergen sensitisation. The most prevalent aero-allergen sensitivities in $A D$ populations are to house dust mite (HDM), pet dander, and pollen. House dust mite avoidance measures have disappointingly failed to demonstrate consistent and clinically significant improvements in $A D$; sufficiently powered HDM immunotherapy trials are lacking. Aero-allergen sensitisation in $A D$ is associated with higher risk of developing asthma and allergic rhinitis; the immunological mechanisms behind this continue to be explored.

\section{Allergic contact dermatitis}

Allergic contact dermatitis manifests when an allergen comes in direct contact with the skin. Although allergens vary in their potential to cause clinical symptoms, patients require prior sensitisation by single or more usually repeated exposure to the substance to become allergic. There is usually a delay of several hours or days 
between exposure and skin symptoms and reactions are typically, but not always, restricted to the area of exposure. The clinical features are those of an eczema (dermatitis): acute redness and swelling of the skin can quickly evolve into a blistering (or vesicular) rash; chronic exposure may lead to dry, thickened areas of skin. Examples of allergic contact dermatitis include nickel sensitivity around jewellery, facial dermatitis from ingredients in cosmetics and hand dermatitis in healthcare workers who wear medical gloves. Patients with suspected allergic contact dermatitis are investigated by patch testing.

\section{Urticaria, angioedema and anaphylaxis}

Urticaria encompasses a heterogeneous group of conditions affecting the skin that share a common clinical presentation of spontaneous, pruritic wheals (or hives). Some $15-25 \%$ of the population will develop urticaria during their lifetime. Acute urticaria can occur as a manifestation of some underlying disorder such as systemic infection, but the condition can also be allergic in origin. An allergic basis for acute urticaria is usually evident from the patient's history and examples include contact urticaria to latex protein found in rubber results and hypersensitivity reactions to penicillin.

About $1.5 \%$ of patients with acute urticaria will proceed to chronic disease, arbitrarily defined as spontaneous and persistent disease of duration of six weeks or more. Around $40 \%$ of patients with chronic urticaria have coexistent angioedema, a deeper swelling of the subcutaneous tissues sharing a common pathophysiology with urticaria at the level of the mast cell. Differential distribution of mast cell populations within the skin and mucosal tissues may account for some of the variation in clinical presentation across the spectrum of urticaria and angioedema.

Although patients with chronic urticaria have a higher than expected incidence of other atopic diseases such as asthma and eczema, the condition is not usually considered allergic in nature. The mainstay of therapeutics for chronic urticaria is usually long-term oral antihistamines; clinically challenging cases requiring offlabel high-dose therapy, sometimes in combination, or the introduction of higher risk strategies such as systemic immunosuppressants. As chronic urticaria may (rarely) be associated with other underlying systemic conditions such as lupus erythematosus or lymphoma, a full history and general physical examination is essential in persistent or severe disease.

Anaphylaxis is a serious systemic allergic disorder characterised by airway and/or cardiovascular compromise. The most serious clinical features of laryngeal oedema, bronchoconstriction and hypotension are typically accompanied by an urticarial rash - in its most severe form anaphylaxis is associated with collapse and rarely death. Foodstuff, medication and venom hypersensitivity are the most common causes of anaphylaxis.

Drug allergy

Hypersensitivity reactions to medications are common and varied. Although a temporal relationship between drug exposure and clinical symptoms is essential, in practice the complexities of polypharmacy and multiple co-morbidities can lead to difficulties in diagnosis. Skin testing is indicated in situations where there is significant clinical doubt and where there is a high risk of subsequent exposure to the drug in question.

\section{Desensitisation}

Although some patients may outgrow, or become tolerant to their allergies, in practice for many patients allergy is lifelong and avoidance of subsequent exposure is the mainstay of treatment. It would be preferable, and indeed it is possible to desensitise patients to their allergies. The basis of immunotherapy is to expose patients to subclinical doses of allergen with the aim of inducing immune tolerance. Immunotherapy is perhaps best described for patients with grass pollen sensitivity, but there are also ongoing trials of immunotherapy for foodstuffs such as peanut allergy. In specific situations such as antibiotic allergy, patients can undergo rapid desensitisation with slowly increasing drug doses until therapeutic levels are tolerated, although allergy will typically return after a course of treatment. 


\section{Highlights}

- Most patients with cutaneous disease, who think they have an allergy, do not.

- Randomly testing patients for allergy is usually unhelpful and, if misinterpreted, may be harmful.

- Skin prick testing is useful in the investigation of immediate (type I) allergic reactions. Patch testing is used to investigate allergic contact dermatitis, a delayed type hypersensitivity reaction. Intradermal testing can be considered for some types of drug hypersensitivity.

- Desensitisation of established allergy is a desirable therapeutic outcome for patients with allergy, but is currently only possible for a limited number of allergens.

- Although the prevalence of allergy is rising, there is a high degree of variability of access to allergy services across healthcare providers, which in turn may lead patients to consider less conventional allergy tests at their own cost.

\section{FURTHER READING}

I Bath-Hextall FJ, Delamere FM, Williams HC. Dietary exclusions for established atopic eczema. Cochrane Database Syst Rev 2008; I:CD005203.

2 Grattan CE, Humphreys F, British Association of Dermatologists Therapy Guidelines and Audit Subcommittee. Guidelines for evaluation and management of urticaria in adults and children. $\mathrm{Br}$ J Dermatol 2007; I57:I I I6-23. http://dx.doi.org/I0.1 I II/j.I3652133.2007.08283.x

3 Joint Task Force on Practice Parameters, American Academy of Allergy, Asthma and Immunology, American College of Allergy, Asthma and Immunology et al. Drug allergy: an updated practice parameter. Ann Allergy Asthma Immunol 2010; 105:259-73. http:// dx.doi.org//0.1016/j.anai.2010.08.002

4 Australasian Society of Clinical Immunology and Allergy. Unorthodox techniques for the diagnosis and treatment of allergy, asthma and immune disorders [Internet]. NSW Australia: ASCIA; 2007 [cited 2013 July 16]. Available from: http://www.allergy.org. au/health-professionals/papers/unorthodox-techniques-fordiagnosis-and-treatment

5 Werfel T, Ballmer-Weber B, Eigenmann PA et al. Eczematous reactions to food in atopic eczema: position paper of the EAACl and GA2LEN. Allergy 2007; 62:723-8. http://dx.doi. org/I0.1III/j.1398-9995.2007.01429.x 


\section{SELF-ASSESSMENT QUESTIONS}

I. A 24-year-old hairdresser presents with troublesome hand eczema that has failed to improve with moderately potent topical steroids. She is worried she may lose her job.

Which ONE of the following would be the most appropriate course of action?

A. Prescribe topical antifungal creams.

B. Treat with a short course of oral steroids.

C. Increase the potency of topical steroids and advise a change of vocation.

D. Prescribe regular emollients, soap substitutes and hand care advice, and refer for patch testing.

E. Refer to a dermatologist for skin prick testing.

2. A 75-year-old man with a history of ischaemic heart disease, chronic airways disease and lateonset atopic dermatitis presents to the Emergency Department with a widespread rash 24 hours after commencing oral penicillin for a chest infection. He is unwell, short of breath with wheeze and has a fever. He is tachycardic, hypotensive and has decreased oxygen saturation.

Which ONE of the following would be the most appropriate course of action?

A. Check his IgE to penicillin esters urgently and await the results before changing treatment.

B. Refer for urgent penicillin desensitisation.

C. Stop his penicillin and commence intravenous cefuroxime.

D. Change his penicillin to a non-beta lactam antiobiotic and refer for an urgent dermatology opinion.

E. Diagnose anaphylaxis to penicillin and administer intravenous adrenaline.

3. A 10-year-old child is brought to her general practitioner with a history of an acute onset itchy, blistering red rash on her left shoulder. She had just returned from a sunny holiday and on the last day had a henna tattoo applied where the skin rash has now appeared.

Which ONE of the following would be the most appropriate course of action?

A. Treat with oral antihistamines and review in one week.

B. Prescribe a potent topical steroid and refer for patch testing.

C. Take a skin biopsy for histological evaluation.

D. Check specific lgG levels to henna.

E. Diagnose henna allergy and give appropriate advice on further avoidance.
4. A young mother brings her three-month-old baby to clinic with a history of eczema since six weeks of age. The baby has never been breastfed and is on a conventional cow's milk protein formula. The mother has concerns about excessive use of topical steroids and is convinced her baby has a food allergy. On examination the patient has widespread, severe eczema and looks a little small for her age.

Which ONE of the following would be the most appropriate course of action?

A. Reassure the mother that allergy is unlikely and prescribe topical therapies for the eczema.

B. Check specific IgE to cow's milk and await the results.

C. Commence oral prednisolone and review in one week.

D. Institute topical therapy and refer to an allergist for further investigation.

E. Diagnose cow's milk protein allergy and change the patient to an amino acid-based formula.

5. A 32-year-old female presents with a threemonth history of recurrent widespread itchy hives across her truck and limbs. She has failed to respond to over-the-counter antihistamines. She is driven to distraction by her itch.

Which ONE of the following would be the most appropriate course of action?

A. Check specific lgE levels to the most common food allergens.

B. Arrange a computed tomography (CT) scan to screen for systemic lymphoma.

C. Refer to a dermatologist for patch testing.

D. Change her antihistamine regimen and review at an early stage.

E. Commence long-term high-dose oral steroids.

This paper was originally published as part of the Dermatology module in the RCPE Online Continuing Medical Education Programme. Online CME, including the anwers to these questions, is available to Fellows and Members at: http://www.rcpe.ac.uk 